\title{
Reconsidering Closure, Underdetermination, and Infallibilism
}

\author{
Jochen Briesen \\ Humboldt-University Berlin
}

\begin{abstract}
Anthony Brueckner $(1994,2005)$ argues for a strong connection between the closure and the underdetermination argument for scepticism. Moreover, he claims that both arguments rest on infallibilism: In order to motivate the premises of the arguments, the sceptic has to refer to an infallibility principle. If this were true, fallibilists would be right in not taking the problems posed by these sceptical arguments seriously. As many epistemologists are sympathetic to fallibilism, this would be a very interesting result. However, in this paper I will argue that Brueckner's claims are wrong: The closure and the underdetermination argument are not as closely related as he assumes and neither rests on infallibilism. Thus even a fallibilist should take these arguments to raise serious problems that must be dealt with somehow.
\end{abstract}

\section{Introduction}

There are a vast number of sceptical arguments that differ along various parameters. Some sceptical arguments make essential use of so-called sceptical hypotheses in order to draw their sceptical conclusion. An argument makes essential use of a sceptical hypothesis, if it is possible to block the argument by ruling out the hypothesis or by knowing that the hypothesis in question is false. I will call arguments of this kind "Cartesian arguments" and the resulting form of scepticism "Cartesian scepticism". There are two promising ways to argue for the sceptical conclusion that we know (almost) nothing about the external world via highlighting a sceptical hypothesis. One route to Cartesian scepticism is based on the closure and the other on the underdetermination principle. These two 
principles lead to different patterns of sceptical arguments: the closure and the underdetermination argument.

With regard to these two arguments Anthony Brueckner defended the following claims:

Claim A. The closure argument presupposes the unterdetermination argument: In order to motivate one of the premises of the closure argument the sceptic has to refer to the underdetermination argument. As a consequence, the closure argument is superfluous in motivating Cartesian scepticism (Brueckner 1994, 830-833; 2005, 388-390).

Claim B. The underdetermination argument and with it the closure argument are based on infallibilism: In order to motivate the premises of the arguments the sceptic has to refer to an infallibility principle. As a consequence, a fallibilist does not have to take these Cartesian arguments to raise a serious challenge (Brueckner 2005, 389-390).

Claim A is interesting because most of the comtemporary discussions of scepticism are focused on the closure argument. If claim A were true, most of the discussions would be concerned with a superfluous argument. And claim B is interesting because most epistemologists are fallibilists. So if claim B were true, for most epistemologists Cartesian scepticism would not pose an interesting problem at all. Cartesian arguments for scepticism are philosophically interesting because their conclusion is very implausible but their premises are at least prima facie very plausible. The challenge is to locate the mistake in these arguments. But if claim B were true and sceptical arguments relied on an infallibility principle as a premise, then - at least for fallibilists - it would be very easy to locate the mistake in these arguments.

However, I will argue that Brueckner's claims A and B are false. The closure argument is not superfluous and neither the closure nor the underdetermination argument rests on infallibilism. Before I give a short overview of the paper, let me make one further remark. This paper is not concerned with the logical relation of the two Cartesian arguments, which has been discussed by Stewart Cohen (1998) and Duncan Pritchard (2005). Both authors agree that the 
closure principle for justification entails the underdetermination principle but they also argue convincingly, contra Anthony Brueckner (1994), that the two principles are not equivalent. ${ }^{1}$ From a logical point of view we are thus faced with two distinct epistemic principles and therefore with two different sceptical arguments that employ these principles, respectively. However, it remains an interesting question, whether these arguments are interrelated in another sense: Is Brueckner right in thinking that the two Cartesian arguments depend on each other and on infallibilism in order to get their premises motivated?

The following discussion of Brueckner's claims A and B is organized as follows. In section 2, I will introduce the closure and the underdetermination argument as well as the principle of infallibilism. In section 3 and 4, I will critically discuss Brueckner's claims A and B and explain why his reasoning is defective. Finally, I will give a short summary in section 4.

\section{Introducing the Arguments and Infallibilism}

In the following arguments $S$ stands for an epistemic subject, $p$ stands for a proposition concerning the external world and $S H$ stands for a sceptical hypothesis that is incompatible with $p$. Let $S H$ be the proposition that we are all brains-in-vats (placed on a deserted planet) with induced hallucinated experiences indistinguishable from our actual experience. ${ }^{2}$

\section{Closure Argument}

(c1) If $S$ knows $p$, then $S$ knows $\neg S H$.

(c2) $S$ does not know $\neg S H$.

(c3) Hence, $S$ does not know $p$.

This argument is called "closure-argument" because premise (c1) is based on the principle that knowledge is closed under known entailment:

\footnotetext{
${ }^{1}$ This seems especially true with regard to the underdetermination principle and the closure principle of knowledge. As it is not even clear whether the closure principle of knowledge entails the underdetermination principle. And it is the closure principle of knowledge with which I am concerned in this paper.

${ }^{2}$ If you think that the brain-in-a-vat scenario is inconsistent with plausible views of semantic externalism, replace the standard brain-in-a-vat scenario by the recent-envatment scenario and restrict the scope of the sceptical argument to knowledge of our current environment.
} 
(CP) For all $S, p, q$, if $S$ knows $p$ and $S$ knows that $p$ entails $q$, then $S$ knows $q .^{3}$

By accepting (CP) premise (c1) can easily be justified because (c1) can be seen as an abbreviated instance of $(\mathrm{CP})$. For the rest of the paper I accept $(\mathrm{CP})$, which I take to be a very plausible principle. In our context premise (c2) is more interesting because it is this premise which Brueckner holds to rest on the underdetermination argument.

What does the underdetermination argument look like? The standard version of the argument is this (see e.g. Brueckner 1994; Pritchard 2005):

\section{Underdetermination Argument}

(u1) If $S$ 's evidence for believing $p$ does not favour $p$ over $S H$, then $S$ 's evidence does not justify $S$ in believing $p$.

(u2) $S$ 's evidence for believing $p$ does not favour $p$ over $S H$.

(u3) Therefore, $S$ lacks justification for believing $p$.

(u4) Hence, $S$ does not know $p$.

This line of thought is called "underdetermination argument" because premise (u1) rests on the underdetermination principle:

(UP) For all $S, p, q$, if $S$ 's evidence for believing $p$ does not favour $p$ over some incompatible hypothesis $q$, then $S$ 's evidence does not justify $S$ in believing $p$.

What is favouring? Following Brueckner I will understand the term " favouring" in the following way. If my evidence favours $p$ over $q$, then $p$ has some epistemic credit which $q$ lacks. In other words: If my evidence favours $p$ over $q$, then it is more reasonable for me to believe $p$ than $q$ (Brueckner 2005, 389).

(UP) is a very plausible principle as well. If my evidence cannot favour $p$ over $q$, then it seems that my evidence can give me at most justification for the

3 John Hawthorne (2003) argues that only a reformulated and weaker version of the closure principle can be defended successfully. If you think this is true, adjust the sceptical premises (c1) and (c2) to this reformulation. It will not undermine the considerations in this paper. 
belief in the disjunction $p$ or $q$. Why should I rationally prefer any one of the disjuncts in this case?

But the argument depends on two more principles. In order to infer (u3) from (u1) and (u2), we have to assume that all justification is evidential, that a lack of appropriate evidence entails a lack of justification - there is no such thing as warrant for nothing or justification by default. Therefore, everybody who endorses the underdetermination argument is committed to the following evidence principle of justification:

(EP) For all $S, p$, if $S$ has justification for believing $p$, then the belief in $p$ is justified by evidence.

And to infer (u4) from (u3), we have to assume that justification is a necessary condition for knowledge, that a lack of justification for the belief that $p$ entails a lack of knowledge that $p$. Therefore, everybody who endorses the underdetermination argument is also committed to the following justification principle of knowledge:

(JP) For all $S$, $p$, if $S$ knows $p$, then $S$ has justification for believing $p$.

By adding two premises to the underdetermination argument that are based on these two principles we would get the full version of the argument. But for simplicity's sake I will focus on the abbreviated version. Even though the two mentioned principles are very controversial, I will accept them for the rest of the paper. With all the mentioned principles in place, the only problematic premise of the underdetermination argument is (u2). It is (u2) which Brueckner holds to rely on infallibilism.

What is infallibilism? Following Brueckner, I will focus on infallibilism with regard to justification. This form of infallibilism holds the following infallibility principle:

(INF) For all $S, p$, if $S$ has justification for believing $p$ in virtue of having evidence $E$, then $E$ guarentees $p$. 
In most of the literature the infallibility principle is formulated with the term "entail" rather than with the term "guarantee". But like Brueckner I try to keep the discussion concerning the relation between Cartesian arguments and infallibilism indifferent with regard to the question whether our evidence is propositional in structure or not. And since an entailment relation can only obtain between propositions or propositional states, I formulated the infallibility principle with the term "guarantee" instead of the term "entail". Whereby " $S$ ' $s$ evidence $E$ guarantees $p$ " is equivalent to " $S$ ' $s$ evidence $E$ entails $p$ or the proposition that $S$ has evidence $E$ entails $p$ ". So the infallibility principle (INF) is a combination of Brueckner's principles (JEP) and (JEP*) (see Brueckner 2005, 384-386). Combining the two principles in one principle only simplifies the discussion a little - nothing substantial hinges on this combination. ${ }^{4}$

In contrast to the closure and underdetermination principle, the infallibility principle (INF) is very implausible - it is incredibly strong and it would make inductive inferences completely unreasonable. With a strong principle like this in place it is easy to argue for a sceptical conclusion. The following sceptical argument is called infallibility argument:

\section{Infallibility Argument}

(i1) If $S$ has justification for believing that $p$ in virtue of having evidence $E$, then $E$ guarentees that $p$.

(i2) $S$ 's evidence $E$ for believing that $p$ does not guarantee $p$.

(i3) Therfore $S$ lacks justification for believing that $p$.

(i4) Hence, $S$ does not know that $p$.

Obviously the infallibility argument just as much rests on (EP) and (JP) as the underdetermination argument does. But in contrast to the underdetermination argument, the infallibility argument also rests on the very implausible infallibility principle (INF). It is thus a bad sceptical argument, which for most

\footnotetext{
${ }^{4}$ Another difference in terminology has to be noted. Brueckner reserves the term "evidential justification" for justification by propositionally structured evidence (believed evidential propositions) (see Brueckner 2005, 386). This is why he refers to non-propositional evidence with "non-evidential justifier". I will use the term "evidence" and "evidential justification" in a more general way, covering both propositional and non-propositional justifiers. Again, nothing substantial hinges on this terminological difference.
} 
epistemologists does not even pose a serious challenge. At least for a fallibilist, who rejects (INF), it is very easy to block the infallibility argument.

\section{Brueckner's Claim A}

Brueckner's claim A is that the closure argument presupposes the unterdetermination argument: In order to motivate one of the premises of the closure argument the sceptic has to refer to the underdetermination argument. Why should we think that claim A is true?

Brueckner argues that the sceptic cannot defend (c2) by referring to Robert Nozick's (1981) sensitivity account of knowledge. Therefore the sceptic has to justify (c2) by referring to the underdetermination argument (Brueckner 2005, $388 ; 1994,828-830)$. Just replace $p$ by $\neg S H$ in the underdetermination argument to see how this might be done.

Why does Brueckner think that (c2) cannot be established via the sensitivity account? Nozick's sensitivity account appeals to the following tracking condition:

(N) If $S$ knows that $p$, then: if $p$ were false, $S$ would not mistakenly believe that $p$.

My belief that $\neg S H$ clearly does not satisfy (N). So, given (N), I do not know $\neg S H$ (see (c2)). But Brueckner takes this strategy for establishing (c2) to be useless to the sceptic, because by appealing to the sensitivity account the sceptic would undermine her justification for premise (c1), which rests on the closure principle (CP). After all, (CP) is false if (N) is true (Brueckner 2005, 388). But this thought is only correct as long as we think sensitivity to be necessary and - possibly together with other conditions uncontroversially met by the beliefs in question sufficient for knowledge. ${ }^{5}$ However, I see no reason why a sceptic should be committed to that. Thus a sceptic who takes sensitivity to be a necessary but not

\footnotetext{
5 That in effect is Nozick's view, which is known as the "sensitivity account of knowledge" (Nozick 1981, 167-188).
} 
sufficient condition for knowledge might very well establish (c2) via the sensitivity principle $(\mathrm{N})$ without being incoherent.

Maybe there is a better reason why the sceptic should not motivate (c2) via sensitivity: The sensitivity account of knowledge is simply wrong - as many counterexamples show, sensitivity is neither necessary nor sufficient for knowledge. However, even if we accepted that the sceptic cannot defend (c2) via $(\mathrm{N})$, we would not be forced to accept Brueckner's conclusion that the sceptic has to appeal to the underdetermination argument in order to motivate (c2). There might be other ways for the sceptic to defend the second premise of the closure argument. Here is a way to justify premise (c2) that is independent of the underdetermination principle and prima facie appealing.

Assume that justification is necessary for knowledge (see the justification principle (JP)) and that basing a belief on evidence is necessary for justification (see the evidence principle (EP)). Now the sceptic can defend (c2) by showing that $\neg S H$ cannot be justified, neither by empirical nor non-empirical evidence.

Why is $\neg S H$ not justifiable by non-empirical evidence? The proposition $\neg S H$ concerns the position of an epistemic subject in the world. And to justify such a proposition you need empirical evidence - you have to take a look at the world to locate your position in it. Thus we might say: $\neg S H$ cannot be justified by non-empirical evidence and therefore we cannot justify $\neg S H$ a priori.

Why is $\neg S H$ not justifiable by empirical evidence? We acquire empirical evidence as a result of an empirical procedure. And this kind of evidence cannot rationally be regarded as any stronger than one's independent reason for supposing that the procedure in question has been executed properly. Therefore, the evidence for the proposition $\neg S H$, that I am not a brain-in-a-vat with hallucinated experiences, cannot rationally be regarded as stronger than my independent reason for thinking that the relevant procedure has been executed properly, hence that it has been executed at all and not just hallucinated to be executed. But that means that justifying $\neg S H$ by empirical evidence already requires that I have justification for the exact same proposition (see Wright 2004, 168). Thus we might say: $\neg S H$ cannot be justified by empirical evidence (for the first time) and therefore we cannot justify $\neg S H$ a posteriori.

The considerations that make (c2) plausible can be summed up thus: ${ }^{6}$

\footnotetext{
${ }^{6}$ See Weatherson (2007) for a related argument.
} 
(1) All evidence is either empirical or non-empirical.

(2) $S$ is not justified in believing $\neg S H$ by non-empirical evidence.

(3) $S$ is not justified in believing $\neg S H$ by empirical evidence.

(4) If $S$ is justified in believing $\neg S H$, then $\neg S H$ is justified by evidence. [based on (EP)]

(5) Therefore, $S$ is not justified in believing $\neg S H$. [from (1), (2), (3), (4)]

(6) If $S$ knows $\neg S H$, then $S$ is justified in believing $\neg S H$. [based on (JP)]

(c2) Hence, $S$ does not know $\neg S H$. [from (5), (6)]

Even though this line of thought is prima facie appealing, it is surely not uncontroversial. $^{7}$ But I did not want to suggest that we should endorse this reasoning. The crucial point is that we have given an argument to defend (c2), which (i) does not refer to the underdetermination principle, (ii) is not utterly implausible and (iii) has been used by some philosophers to argue for the sceptical premise (c2). Thus we can conclude: Brueckner's claim A is false and the closure argument is not superfluous in motivating Cartesian scepticism.

Notice that the given reasoning in favour of (c2) forces the sceptic to accept the two additional principles on which the underdetermination argument were based: the justification principle (JP) and the evidence principle (EP).

\section{Brueckner's Claim B}

Brueckner's Claim B is that the underdetermination argument and with it the closure argument are based on infallibilism: In order to motivate the premises of the arguments the sceptic has to refer to an infallibility principle. Brueckner motivates claim B by showing that the underdetermination argument, when used to argue for the second premise (c2) of the closure argument, presupposes

\footnotetext{
${ }^{7}$ Some semantic externalists and defenders of transcendental arguments of other kind would not accept (2). Some epistemic externalists with regard to justification and dogmatists would not accept (3). Other epistemic externalists and defenders of the view that there is something like warrant for nothing or justification by default would not accept (4). And epistemic externalists with regard to knowledge would not accept (6). The fact that all the important antisceptical strategies in the literature are so easily mapped on the argument speaks in favour of my view, that something like this argument lies at the heart of the sceptics motivation of (c2).
} 
infallibilism. And via claim A he infers that the closure argument in general is based on infallibilism. If this reasoning were correct, then the sceptic would need infallibilism to motivate her sceptical line of thought. And since fallibilism about knowledge and justification is a widely held view in epistemology, many philosophers were right in not taking Cartesian scepticism seriously. But we have already seen that claim $\mathrm{A}$ is false, and this is the reason why the second step of Brueckner's consideration is defective. The closure argument is not based on infallibilism because our motivation of (c2) was neither based on the underdetermination nor on the infallibility principle.

Before we turn to the relation of the underdetermination argument and infallibilism let me make one further remark. It might be true that the closure argument along with our motivation for (c2) entails the infallibility principle (INF). But of course this does not mean that a fallibilist is able to sidestep sceptical worries. The fact that the sceptical premises can be used to argue for implausible theses was clear all along. After all, the sceptical premises entail that we know almost nothing about the external world. That the prima facie plausible premises of the closure argument also entail the very implausible infallibilty principle should be even more worriesome for the fallibilist - it is surely not a free ticket to ignore sceptical arguments. As long as the sceptic does not use the infallibility principle in order to motivate her premises, the closure argument raises a serious problem even (or especially) for fallibilists.

But what about the underdetermination argument and its relation to infallibilism? As mentioned before, Brueckner only shows that the underdetermination argument relies on infallibilism, when used as a motivation for (c2) (Brueckner 2005, 388-390). But his line of thought can easily be reconstructed to argue that the underdetermination argument in general is based on infallibilism.

\section{Underdetermination Argument}

(u1) If $S$ 's evidence for believing $p$ does not favour $p$ over $S H$, then $S$ 's evidence does not justify $S$ in believing $p$. [based on (UP)]

(u2) $S$ 's evidence for believing $p$ does not favour $p$ over $S H$.

(u3) Therefore, $S$ lacks justification for believing $p$. [from (u1), (u2), (EP)]

(u4) Hence, $S$ does not know $p$. [from (u3), (JP)] 
The crucial premise in our context is $(\mathrm{u} 2)$. Brueckner tries to argue that in order to establish (u2), we finally have to refer to the infallibility principle. The first step in the envisaged motivation for $(\mathrm{u} 2)$ is what Brueckner calls the "sameness of evidence lemma":

(SEL) One has exactly the same evidence in the good case and in the bad case. $^{8}$

The good case is that in which $\mathrm{p}$ is true and $S H$ is false and the bad case is that in which $S H$ is true and $p$ false. Brueckner accepts that it is reasonable to think that (u2) follows from (SEL) (Brueckner 2005, 389). ${ }^{9}$ As far as I can see (SEL) is plausible. As long as we think of our evidence for $p$ as our perceptual evidence, and do not take a disjunctivist view on perception or Timothy Williamson's (2000) view on evidence, (SEL) seems to follow from the description of $S H$ alone. ${ }^{10}$ For example, take our perceptual evidence to be our seemings. Then my evidence for the belief that there is a table in front of me is that it seems to me that there is a table in front of me. And of course it would seem to me that there is a table in front of me if $S H$ were realized - this is how the sceptical hypothesis is designed. So (SEL) follows from the description of $S H$ along with plausible views on perceptual evidence.

But Brueckner thinks that in espousing (SEL), the sceptic is calling attention to the (alleged) fact that it is possible that my evidence $E$ for $p$ should be

\footnotetext{
${ }^{8}$ Brueckner eventually speaks of the "sameness of justifier lemma" (SJL), because he reserves the term " evidence" for justifiers with propositional content. I will use the term "evidence" to cover both propositional and non-propositional justifiers (see fn:4).

9 Note that Brueckner refers to the second premise of the underdetermination argument (u2) with " F".

${ }^{10}$ Of course other accounts of perceptual evidence that would be incompatible with (SEL) are logically possible. But the most plausible and recently defended views of this sort are disjunctivism and Williamson's view on evidence. On a disjunctivist view the intrinsic nature of a perceptual state is determined by the perceived object. This is why a disjunctivist thinks that the perceptual states that I have when perceiving a fish and when merely halluzinating a fish are not tokens of a single perceptual-state-type, even though we cannot tell the difference from a first persons perspective. Therefore they think that our perception in the good p-case and in the bad SH-case are very different and hence that we cannot have the same evidence in the two cases. Timothy Williamson (2000) on the other hand holds the view that the body of our evidence is the body of our knowledge. As long as you think that the body of knowledge of a peson in a situation where $\mathrm{p}$ is realized differs from the body of knowledge of a person in a situation where $\mathrm{SH}$ is realized, it follows that we cannot have the same evidence in the good p-case and in the bad SHcase. But of course both of the mentioned views are very controversial.
} 
present when $S H$ is true - in other words, the proposition that I have evidence $E$ for $p$ is consistent with $S H$ and, concomitantly, with the denial of $p$ - in other words, my evidence $E$ for $p$ fails to guarentee $p$. Thus in espousing (SEL) the sceptic is calling attention to the fact that my evidence $E$ does not guarentee $p$ (Brueckner 2005, 390). Brueckner takes the upshot of this observation to be: The sceptic's strategy of using (UP), (u2) and (SEL) to argue for (u3) (that $S$ lacks justification for $p$ ) is simply a use of the infallibility principle (INF) in arguing for (u3) (Brueckner 2005, 390). ${ }^{11}$

It is not easy to understand Brueckner's line of thought. I do not really see why Brueckner's reconstruction of the sceptic's strategy would amount simply to a use of the infallibility principle (INF). The principle (INF) just did not arise in his reconstruction. But instead of speculating what exactly Brueckner has in mind, I will do the following. According to Brueckner, the sceptic's reliance on infallibilism is somehow due to the motivation of (u2) via (SEL) and the interrelation of (UP) and (SEL). So in order to sidestep Brueckner's worries altogether, we should find a way to motivate the crucial second premise (u2) of the underdetermination argument without referring to the sameness of evidence lemma (SEL). I will present two promising principles that can be used for that purpose.

The first principle is the explanation principle:

(EXP) For all $S, p, q$, if $q$ causally explains $S$ 's evidence $E$ (at least as good as $p$ does), then $E$ cannot favour $p$ over the incompatible alternative $q$.

The explanation principle (EXP) is at least prima facie very plausible. The principle is much weaker than (INF) and it allows that we can evidentially favour

\footnotetext{
${ }^{11}$ Here is a quote of the relevant passage: "In espousing SJL, the skeptic is calling attention to the (alleged) fact that it is possible that my putative experiential justifier for $\sim$ SK should be present when SK is true. In other words, the proposition that I have the putative experiential justifier for $\sim \mathrm{SK}$ is consistent with SK and, concomitantly, with the denial of $\sim \mathrm{SK}$. In other words, the proposition that I have the putative experiential justifier for $\sim$ SK fails to entail $\sim$ SK. Sound familiar? The upshot is that the skeptic's strategy of using SJL, $\sim F$, and UP to establish his premise 2 is simply a use of the entailment principle JEP* to show a lack of justification for $\sim$ SK." (Brueckner 2005, 390) For an explanation of the terminological differences see fn. 4, 8, 9, and keep in mind that I reconstructed Brueckner's thought to argue that the underdetermination argument in general is based on infallibilism.
} 
some possibilities over others by induction. But it is strong enough to establish (u2):

(1*) If $S H$ causally explains $S$ 's evidence $E$ (at least as good as $p$ does), then $E$ cannot favour $p$ over $S H$. [based on (EXP)]

(2*) $S H$ causally explains $E$ (at least as good as $p$ does).

(u2) Therefore, $S$ 's evidence cannot favour $p$ over $S H$.

Of course assumption $\left(2^{*}\right)$ is controversial. But as many failed antisceptical attempts based on the inference to the best explanation illustrate, it is very hard to argue for the view that the explanation of our experience by the sceptical hypotheses is worse than our standard explanation. ${ }^{12}$

The second principle I want to present is the entailment principle:

(ENT) For all $S, p, q$, if $q$ entails the proposition that $S$ has evidence $E$, whereas the incompatible alternative $p$ does not entail the proposition that $S$ has evidence $E$, then $E$ cannot favour $p$ over $q$.

In my view principle (ENT) seems even more plausible than (EXP). Let $S$ 's evidence $E$ consist in $S$ 's many perceptual experiences of white swans, and let this evidence $E$ be the only evidence she has got for the beliefs in the following propositions. Let $w$ stand for the proposition that most swans are white and $b$ for the proposition that most swans are black. Neither $w$ nor $b$ entail the proposition that $S$ has evidence $E$. Therefore, the principle (ENT) allows that $S$ 's evidence can favour $w$ over the incompatible alternative $b$. Thus the principle is weak enough to allow that we evidentially favour some beliefs over others by induction. Now let $b^{*}$ stand for the proposition that most swans are black but $S$ only perceived white ones. It is intuitively correct to think that $S$ 's evidence $E$, which solely consists in her perception of white swans, cannot favour $w$ over $b^{*}$. And this is exactly what principle (ENT) predicts: $b^{*}$ entails that $S$ has evidence $E$, whereas $w$ does not,

\footnotetext{
${ }^{12}$ For an interesting antisceptical attempt to block the sceptical argument via an inference to the best explanation see Vogel (1990).
} 
hence $S$ 's evidence $E$ cannot favour $w$ over the incompatible alternative $b^{*} .^{13}$ Now, by accepting (ENT) it is easy to establish (u2):

(1**) If $S H$ entails the proposition that $S$ has evidence $E$, whereas $p$ does not entail the proposition that $S$ has evidence $E$, then $E$ cannot favour $p$ over $S H$. [based on (ENT)]

(2**) $S H$ entails the proposition that $S$ has evidence $E$ and $p$ does not.

(u2) Therefore, $S$ 's evidence cannot favour $p$ over $S H$.

Of course (2**) is controversial. But again, as long as we think of our evidence $E$ as our perceptual evidence and do not take a disjunctivist view on perception or Williamson's view on evidence, $\left(2^{* *}\right)$ looks very plausible as well (see fn. 9).

Note that I do not mean to suggest that we should endorse the arguments that establish (u2) via (EXP) and (ENT). Even though I take the arguments to be at least prima facie very plausible, both arguments are surely controversial. The crucial point is that we have given two arguments to establish (u2) with the follwing characteristics. First, the arguments are at least as plausible as Brueckner's motivation for (u2) via (SEL). Second, the arguments are not referring to the sameness of evidence lemma (SEL), which in Brueckner's view is somehow responsible for the sceptic's dependence on infallibilism in motivating (u2). Third, the arguments in favour of (u2) we have presented are not relying on the infallibility principle - the principles (EXP) and (ENT) are weaker than an analogous infallibility principle and thus more plausible, but they are strong enough to motivate the second premise of the underdetermination argument (u2). Thus we can conclude: Brueckner's claim B is false - neither the closure nor the underdetermination argument is based on infallibilism. A fallibilist should accept that these arguments raise a serious a challenge that must be answered somehow.

Again, it might be true that the underdetermination argument together with our motivation of ( $\mathrm{u} 2$ ) entail the infallibility principle (INF). But as with regard to the closure argument, this is surely not a free ticket for fallibilists to ignore the underdetermination argument. As long as the sceptic does not refer to (INF) in

\footnotetext{
${ }^{13}$ Remember that we only allowed $S$ 's perceptual experiences of white swans as $S$ 's evidence for $w$ - no background assumptions whatsoever are in place as additional evidence.
} 
establishing her premises, the underdetermination argument raises a challenge even (or especially) for fallibilists.

\section{Summary}

Brueckner's claim A is that the closure argument rests on the underdetermination argument: In order to motivate the second premise (c2) of the closure argument the sceptic has to refer to the underdetermination argument. I have shown that claim A is false. I have given a plausible argument in favour of (c2) without referring to the underdetermination principle. Thus the closure argument is not superfluous in motivating Cartesian scepticism.

Brueckner's claim B is that the underdetermination argument and with it the closure argument rest on infallibilism: In order to establish their premises the sceptic has to refer to the infallibility principle (INF). My answer to claim A already shows that the second part of claim B is not true - the closure argument does not rest on infallibilism because our motivation of (c2) is neither relying on the underdetermination nor on the infallibility principle.

What about the underdeterminaton argument? A presupposition of Brueckner's reasoning why the underdetermination argument eventually rests on infallibilism is that the scpetic has to refer to the sameness of evidence lemma (SEL) in order to motivate the second premise of the underdetermination argument (u2). I have presented two ways to motivate (u2) without referring to (SEL) or the infallibility principle (INF). Therefore, the underdetermination argument does not rest on infallibilism, and even a fallibilist has to take the arguments to raise a serious challenge that must be answered somehow. ${ }^{14}$

\section{References}

Brueckner, A (1994). 'The Structure of the Skeptical Argument', Philosophy and Phenomenological Research 54, 827-834.

\footnotetext{
14 Thanks to Anthony Brueckner, Martin Smith, Brian Weatherson, and Elia Zardini for helpful comments and discussions on the material.
} 
Brueckner, A (2005). 'Fallibilism, Underdetermination, and Skepticism', Philosophy and Phenomenological Research 71, 384-391.

Cohen, S. (1998). 'Two Kinds of Skeptical Arguments', Philosophy and Phenomenological Research 58, 143-159.

Hawthorne, J. (2003). 'The Case for Closure', in M. Steup and E. Sosa (eds.), Contemporary Debates in Epistemology, Oxford: Blackwell Publishing.

Nozick, R. (1981). Philosophical Explanations, Cambrige: Harvard University Press.

Pritchard, D. (2005). 'The Structure of Sceptical Arguments', The Philosophical Quarterly 55, 3752.

Stroud, B. (1984). The Significance of Philosophical Scepticism, Oxford: Oxford University Press.

Vogel, J. (1990): 'Cartesian Skepticism and Inference to the best Explanation', Journal of Philosophy 87, 658-666.

Weatherson, B. (2007). 'The Bayesian and the Dogmatist', Proceedings of the Aristotelian Society 107, 169-185.

Williamson, T. (2000). Knowledge and its Limits, Oxford: Oxford University Press.

Wright, C. (2004): 'Warrant for Nothing: Notes on Epistemic Entitlement', Proceedings of the Aristotelian Society, Supplementary Volume 78, 167-212. 